\title{
BIODIVERSIDADE FLAVONOÍDICA E ASPECTOS FARMACOLÓGICOS EM ESPÉCIES DOS GÊNEROS Ouratea E Luxemburgia (OCHNACEAE)
}

\author{
Luciano R. Suzart, Juliana F. de S. Daniel e Mário G. de Carvalho* \\ Departamento de Química, Instituto de Ciências Exatas, Universidade Federal Rural do Rio de Janeiro, Antiga Rodovia Rio- \\ São Paulo, BR 465, km 7, 23890-000 Seropédica - RJ, Brasil \\ Maria Auxiliadora Coelho Kaplan \\ Núcleo de Pesquisa de Produtos Naturais, Universidade Federal do Rio de Janeiro, Rio de Janeiro - RJ, Brasil
}

Recebido em 31/7/06; aceito em 9/11/06; publicado na web em 5/6/07

\begin{abstract}
FLAVONOIDIC BIODIVERSIY AND PHARMACOLOGIC ASPECTS IN THE SPECIES OF THE Ouratea AND Luxemburgia GENERA (OCHNACEAE). This work describes the flavonoids and biflavonoids found in species of Ouratea and Luxemburgia as chemical markers that serve to detect the difference between these taxa according to the linkage between the flavonoidal units. A rational nomenclature is proposed and the pharmacological potencial is discussed.
\end{abstract}

Keywords: Ochnaceae; Ouratea; Luxemburgia.

\section{INTRODUÇÃO}

A família Ochnaceae pertence à ordem Theales ${ }^{1}$ e compreende cerca de 28 gêneros e 400 espécies de ampla distribuição nas regiões tropicais e subtropicais de todo o mundo. No Brasil, ocorrem aproximadamente 9 gêneros com 105 espécies $^{2}$. São plantas essencialmente arbóreas ou arbustivas. As espécies espalhadas pelo país recebem designações específicas como Angelim (Ouratea vaccinoides), Caju Bravo (Ouratea floribunda e Ouratea salicifolia) e Coração de Bugre (Ouratea parviflora). Ouratea floribunda e Ouratea castanaefolia são empregadas em ornamentação urbana. No Nordeste, as espécies desse gênero são conhecidas como batiputá3.

As espécies de Ochnaceae são capazes de biossintetizar flavonóides e biflavonóides, sendo a família mais bem representada pelos gêneros Ouratea, Luxemburgia, Ochna e Lophira ${ }^{47}$. A frequiência e a diversidade estrutural dos biflavonóides em espécies desses gêneros permitem que sejam utilizados como marcadores taxonômicos.

Os biflavonóides constituem uma classe de flavonóides diméricos, diferenciando-se de outros oligômeros como as proantocianidinas, devido à origem biogenética das unidades constituintes. A maioria dos representantes dessa classe de produtos naturais é formada pelos dímeros flavona-flavona, flavona-flavanona, flavanona-flavanona além de ocorrerem, mais raramente, os dímeros de chalconas e de isoflavonas. Quando as duas unidades são iguais, constituem os bisflavonóides e quando as duas unidades são diferentes, os biflavonóides. As ligações entre as unidades flavonoídicas podem ser C-C ou C-O-C envolvendo os anéis A, B ou C dos monômeros (Figura 1). Raramente ocorre alteração no padrão de oxigenação dos precursores, sendo garantida a oxigenação em 5, 7 e 4' e raramente uma oxidação adicional em 3'. Podem ocorrer oxidações nas posições 6,8 ou $3^{\prime}$ e quando isso acontece é, certamente, proveniente da outra unidade ligada nessa posição via C-O-C (34, Figura 2).

A primeira biflavona, foi isolada em 1929, e é conhecida como gingentina. Desde então, mais de mil biflavonóides foram isolados de plantas e muitas atividades biológicas têm sido relacionadas a essa classe de substâncias ${ }^{8}$.

Utiliza-se a numeração dos biflavonóides atribuindo números

*e-mail: mgeraldo@ufrrj.br ordinários para os anéis A e C e primados (') para o anel $\mathbf{B}$ de um dos monômeros. Para a segunda unidade, empregam-se números ordinários duplamente primados (") para os núcleos $\mathbf{A}$ e $\mathbf{C}$ e números ordinários triplamente primados ("') para o núcleo B. De acordo com os átomos de carbonos envolvidos na ligação entre as unidades, os dímeros são classificados em grupos de biflavonóides (Figura 1) ${ }^{9-11}$, além dos dímeros de chalconas: C-3 $\rightarrow$ O-C-4", (luxenchalconas) $^{12}$ e C-3' $\rightarrow$ C $3^{\prime \prime}$ (brackeninas) ${ }^{13}$ e dos dímeros de isoflavonas C-2 $\rightarrow$ C-2" (hexaspermonas) ${ }^{14}$, sem destacar os dímeros com duas ligações entre as unidades. Certos grupos hidroxila podem apresentar-se metilados, originando os respectivos éteres metílicos que, às vezes, recebem nomes especiais ${ }^{15-18}$.

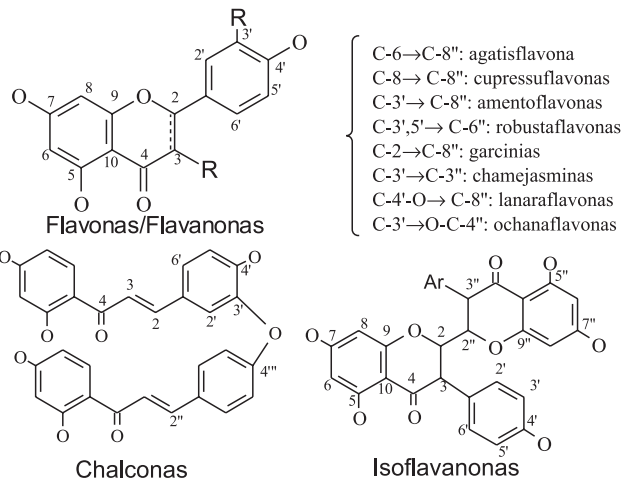

Figura 1. Dímeros de flavonóides

Nos casos dos gêneros Ouratea e Luxemburgia, além de alguns monômeros, tem-se detectado com freqüência a presença de bi- ou bisflavonóides, destacando-se as hexaspermonas (4-6), amentoflavonas $(\mathbf{1 1}, \mathbf{1 8})$, agatisflavonas (12) e robustaflavona (24), lanaraflavonas $(\mathbf{1 5 - 1 7}, \mathbf{3 4})$ em Ouratea, Figura 2. Em Luxemburgia foram detectados os biflavonóides derivados de chalconas, luxenchalcona (25, C-3' $\rightarrow$ O-C-4'”) e ochnaflavona (20, também $\mathrm{C}-3$ ' $\rightarrow \mathrm{O}-\mathrm{C}-4$ '"'), Figura 2, que podem ter como precursor a luxenchalcona. Isso permite perceber a diferença entre esses gêneros sendo que Luxemburgia é próximo ao gênero Ochna e metaboliza com frequiência os dímeros de chalconas ${ }^{19}$. Por outro lado, Ouratea tem tendência em metabolizar com mais frequiência os dímeros de 
flavonas. A ocorrência de biflavonóides nos gêneros Ouratea e Luxemburgia permite destacar a importância da química dos mesmos como potencial farmacológico e considerar as substâncias dessa classe como marcadores quimiotaxonômicos.

\section{FLAVONÓIDES IDENTIFICADOS NOS GÊNEROS Ouratea E Luxemburgia}

Além dos flavonóides e biflavonóides, esses gêneros são bioprodutores de outras classes de metabólitos como triterpenos, diterpenos, depsídeos, ésteres graxos e triglicerídeos ${ }^{17,20-23}$. Entre essas classes de substâncias, os biflavonóides recebem destaque na literatura, devido à freqüência e abundância com que são encontrados nesses gêneros e cuja diversidade estrutural é devida, principalmente, aos diferentes padrões de ligações entre seus monômeros.

Do gênero Ouratea foram isolados biflavonóides dos grupos amentoflavona $(\mathbf{1 1}, \mathbf{1 8})$, agatisflavona (35), bigenkanina $(\mathbf{8})$, lanaraflavona (15) e seus derivados, além de outros dímeros como lofironas (32), calodeninas $(28,31)$ e flavumonas $(29,30)$. A junção de isoflavanonas $(\mathrm{C}-2 \rightarrow \mathrm{C}-2$ ") deu origem às hexaspermonas
A, B e C $(4 ; 5 ; \mathbf{6})$, respectivamente. A ligação tipo C-O-C é característica das ochnaflavonas (C-3' $\rightarrow \mathrm{O}-\mathrm{C}-4$ "') e das lanaraflavonas $(\mathrm{C}-4$ '-O $\rightarrow$ C-8"), sendo o grupo das ochnaflavonas (20) que ocorrem em espécies de Luxemburgia ${ }^{12,24}$ e Ochna $^{6}$. Desse gênero foram isoladas as chalconas $(\mathbf{2 2}, \mathbf{2 3})$ e a bichalcona (C-3 $\rightarrow$ O-C-4", 25), Figura 2.

Uma característica do gênero Ouratea é a ligação entre seus monômeros, sendo as mais abundantes C-3 $\rightarrow$ C-8" e C-6 $\rightarrow$ C-8", acrescentando uma variação no padrão de metilação. No caso dos derivados da agatisflavona (C-6 $\rightarrow$ C-8") a metilação é mais freqüente na posição 7 (10). Com ligações (C-O-C), foram isolados os dímeros (C-4'-O $\rightarrow$ C-8" ) e (C-3' $\rightarrow$ O-C-4"'), incluindo a ligação entre os monômeros das bichalconas, que são característicos do gênero Luxemburgia ${ }^{12,24}$, enquanto que de Ouratea se isolaram os derivados de lanaraflavona (C-4'-O-C-8") com diferentes padrões de metilação $(\mathbf{1 6}, \mathbf{1 7}, \mathbf{3 4})$. A forma de ligação e os tipos de monômeros podem ser usados como diferenciadores entre esses gêneros. A diferença principal entre ambos é que em Ouratea a biflavona mais abundante é a 7"- metilagatisflavona (10) e em Luxemburgia, a diidroochnaflavona (20), Figura 2.
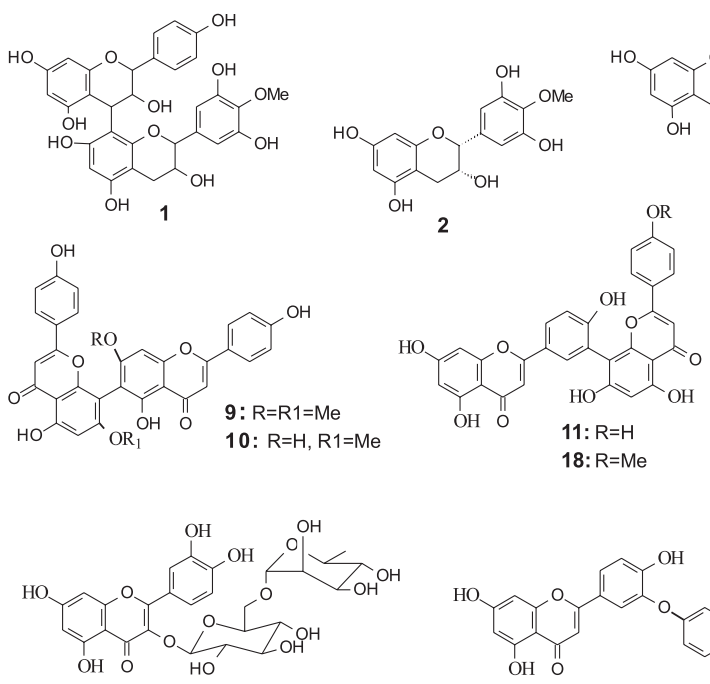

19<smiles>[Z6]Oc1c(-c2cc(-c3cc(=O)c4c(O)cc(O)cc4o3)ccc2O)c(O)cc2oc(-c3ccc(O)cc3)cc(=O)c12</smiles><smiles>O=C1CC(c2ccc(O)cc2)Oc2cc(O)c3c(C(=O)c4ccc(O)cc4O)c(-c4ccc(O)cc4)oc3c21</smiles><smiles>COc1cc(C2Oc3cc(O)ccc3C[C@H]2O)cc(O)c1O</smiles>

3

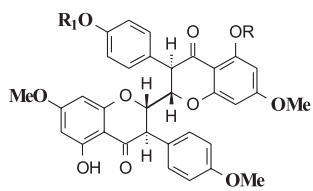

4: $\mathrm{R}=\mathrm{H}, \mathrm{R}_{1}=\mathrm{Me} \quad$ 5: R=Me, $\mathrm{R}_{1}=\mathrm{H}$ 6: $R=R_{1}=H$

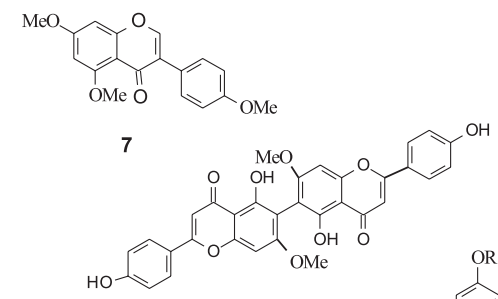<smiles>[Z12]C(=O)c1c(O)cc(OC)c2c1OC(c1ccc(OC)c(OC)c1)C(O)C2=O</smiles>

12<smiles>Oc1ccc(C2Oc3cc(O)cc(O)c3CC2O)cc1</smiles><smiles>[R]c1cc(-c2coc3c(Cl)c(OC)c(Cl)c(O)c3c2=O)cc(Cl)c1O</smiles>

13: $\mathrm{R}=\mathrm{H}$ 14: $\mathrm{R}=\mathrm{Cl}$
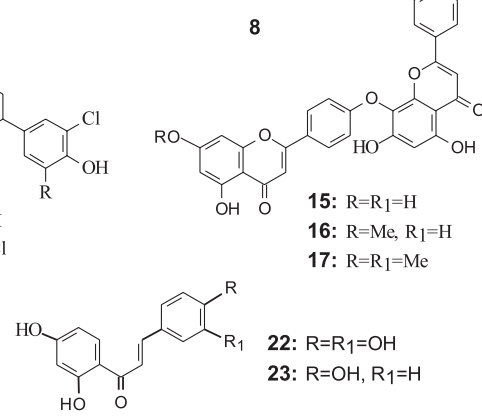<smiles>O=C(/C=C/c1ccc(Oc2cc(/C=C/C(=O)c3ccc(O)cc3O)ccc2O)cc1)c1ccc(O)cc1O</smiles><smiles>Oc1ccc([C@H]2Oc3cc(O)ccc3[C@@H](c3c(O)cc(O)c4c3O[C@H](c3ccc(O)cc3)[C@H](O)[C@H]4O)[C@H]2c2ccccc2)cc1</smiles>

31
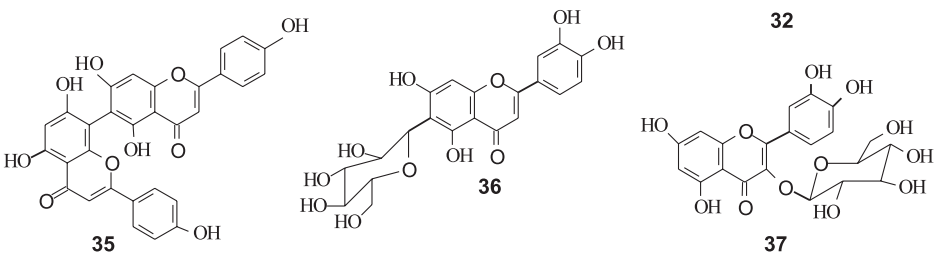<smiles>[R20]c1cc(O)c2c(=O)cc(-c3ccc(O)c(O)c3)oc2c1C(CO)OC(O)C(O)CO</smiles>

$\mathrm{R}=\mathrm{H}$ 27: $\mathrm{R}=\mathrm{CH}_{3}$<smiles>CC(C(=O)c1ccc(O)cc1O)c1coc2cc(O)ccc2c1=O</smiles><smiles>Oc1ccc(Cc2ccc(O)cc2)cc1</smiles><smiles></smiles>

28: $\mathrm{R}=\mathrm{R}_{1}=\mathrm{H}$ 29: $\mathrm{R}=\mathrm{H}, \mathrm{R}_{1}=\mathrm{OH}$

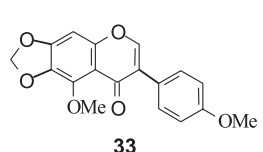

33

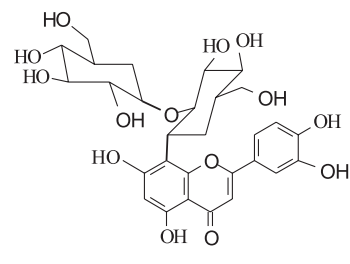

38

Figura 2. Estruturas dos flavonóides isolados de Ouratea e Luxemburgia 
Tabela 1. Ocorrência de flavonóides em espécies de Ouratea e Luxemburgia (Ochnaceae)

\begin{tabular}{|c|c|c|}
\hline Flavonóides & Espécies & Ref. \\
\hline proantocianidina $(\mathbf{1})$ & Ouratea sp. & 32 \\
\hline catequina $(2)$ & Ouratea sp. & 32 \\
\hline cianidina $(\mathbf{3})$ & O. affinis Engl. & 33 \\
\hline cianidina $(3)$ & O. calantha Gilg. & 33 \\
\hline $\begin{array}{l}\text { 5-OH-4',7-OMe-2,3-trans-isoflavonona }\left(2 \rightarrow 2^{\prime \prime}\right)-5^{\prime \prime}-\mathrm{OH} 4^{\prime \prime \prime} 7^{\prime \prime}- \\
\left.\text { OMe-2", } 3^{\prime \prime} \text {-trans-isoflavanona. (hexaspermona } \mathrm{A}, \mathbf{4}\right)\end{array}$ & O. hexasperma (St. Hil.) Bail & 14 \\
\hline $\begin{array}{l}\text { 5-OH-4',7- OMe-2,3-trans-isoflavanona }\left(2 \rightarrow 2^{\prime \prime}\right)-4^{\prime \prime \prime} \mathrm{OH} 5^{\prime \prime}, 7^{\prime \prime}- \\
\text { OMe-2" } 3^{\prime \prime} \text {-trans-isoflavanona. (hexaspermona B,5) }\end{array}$ & O. hexasperma (St. Hil.) Bail & 14 \\
\hline $\begin{array}{l}\left.\text { 5-OH-4',7-OMe-2,3-trans-isoflavanona-( } 2 \rightarrow 2^{\prime \prime}\right)-4^{\prime \prime \prime}, 5^{\prime \prime}-\mathrm{OH}-7^{\prime \prime}- \\
\mathrm{OMe}-2^{\prime \prime}, 3^{\prime \prime} \text {-trans-isoflavanona. (hexaspermona C,6) }\end{array}$ & O. hexasperma (St. Hil.) Bail & 14 \\
\hline $5,7,4^{\prime}$-OMe-isoflavona $(7)$ & O. hexasperma (St. Hil.) Bail & 14 \\
\hline$\left(6 \rightarrow 6^{\prime \prime}\right)$-bigenkanina $(\mathbf{8})$ & O. spectabilis (Mart.) Engl. & 16 \\
\hline $\begin{array}{l}4^{\prime}, 5-\mathrm{OH}-7 \text {-metoxiflavona }(6 \rightarrow 8) 5^{\prime \prime}, 4^{\prime \prime \prime}-\mathrm{OH}-7^{\prime \prime}-\mathrm{OMe} \\
\text { flavona }\left(7,7^{\prime \prime} \text {-O-dimetilagatisflavona,9) }\right.\end{array}$ & O. spectabilis (Mart.) Engl. & 16 \\
\hline $\begin{array}{l}4,5,7-\text { OH-flavona }\left(6 \rightarrow 8^{\prime \prime}\right) 5^{\prime \prime}, 4^{\prime \prime \prime}-\mathrm{OH}-7^{\prime \prime}-\mathrm{OMe} \text { flavona } \\
\left(7^{\prime \prime}-\mathrm{O}-\mathrm{metilagatisflavona,10}\right)\end{array}$ & O. hexasperma (St. Hil.) Bail & 15 \\
\hline $\begin{array}{l}\text { 4',5,7-OH-flavona- }\left(3^{\prime} \rightarrow 8^{\prime \prime}\right)-4^{\prime \prime \prime}, 5^{\prime \prime}, 7^{\prime \prime}-\text { OH flavona. } \\
\text { (amentoflavona,11) }\end{array}$ & O. multiflora Pohl & 34 \\
\hline $\begin{array}{l}\left.\text { 3-OH-4',5,7-OMe-flavona-( } 6 \rightarrow 8^{\prime \prime}\right)-3^{\prime \prime}-\mathrm{OH}-3^{\prime \prime \prime}, 4^{\prime \prime \prime}, 5^{\prime \prime}, 7^{\prime \prime}- \\
\text { OMe flavona, } 12\end{array}$ & O. multiflora Pohl & 34 \\
\hline $3^{\prime}, 6,8$-cloro-4',5-OH-7-OMe isoflavona $(\mathbf{1 3})$ & O. semisserata (Mart.) Engl & 17 \\
\hline $3^{\prime}, 5^{\prime}, 6,8$-cloro-4',5-OH-7-OMe isoflavona (14) & O. semisserata (Mart.) Engl & 17 \\
\hline 5,7-OH-flavona-( $\left.4^{\prime} \rightarrow \mathrm{O} \rightarrow 8^{\prime \prime}\right)-4^{\prime \prime \prime}, 5^{\prime \prime}, 7^{\prime \prime}-\mathrm{OH}$ flavona $(\mathbf{1 5})$ & O. semisserata (Mart.) Engl & 17 \\
\hline 5-OH -7- OMe-flavona- $\left(4^{\prime} \rightarrow \mathrm{O} \rightarrow 8^{\prime \prime}\right)-4^{\prime \prime \prime}, 5^{\prime \prime}, 7^{\prime \prime}-\mathrm{OH}$ flavona $(\mathbf{1 6})$ & O. semisserata (Mart.) Engl & 17 \\
\hline 5-OH-7-OMe-flavona- $\left(4^{\prime} \rightarrow \mathrm{O} \rightarrow 8^{\prime \prime}\right)-5^{\prime \prime}, 7^{\prime \prime}-\mathrm{OH}-4^{\prime \prime \prime}-\mathrm{OMe}$ flavona $(\mathbf{1 7})$ & O. semisserata (Mart.) Engl & 17 \\
\hline $\begin{array}{l}\text { 4',5,7-OH-flavona- }\left(3^{\prime} \rightarrow 8^{\prime \prime}\right)-4^{\prime \prime \prime}, 5^{\prime \prime}, 7^{\prime \prime}-\mathrm{OH} \text { flavona. } \\
\text { (amentoflavona,11) }\end{array}$ & O. semisserata (Mart.) Engl & 17 \\
\hline $\begin{array}{l}4^{\prime} 5,7-\mathrm{OH}-\mathrm{flavona}-\left(3^{\prime} \rightarrow 8^{\prime \prime}\right)-5^{\prime \prime}, 7^{\prime \prime}-\mathrm{OH}-4^{\prime \prime \prime}-\mathrm{OMe} \text { flavona } \\
\text { (podocarpusflavona,18) }\end{array}$ & O. semisserata (Mart.) Engl & 17 \\
\hline quercetina 3 -O- $\alpha$-L-raminosil $(1 \rightarrow 6)$-b-D-glicopiranosil (rutina, 19) & O. semisserata (Mart.) Engl & 17 \\
\hline quercetina 3 -O- $\alpha$-L-raminosil $(1 \rightarrow 6)$-b-D-glicopiranosil (rutina, 19) & Luxemburgia nobilis (Eichl) & 24 \\
\hline $4^{\prime}, 5,7-\mathrm{OH}$-flavona- $\left(3^{\prime} \rightarrow \mathrm{O} \rightarrow 4^{\prime \prime \prime}\right)-5^{\prime \prime}, 7^{\prime \prime}-\mathrm{OH}$ flavanona $(\mathbf{2 0})$ & Luxemburgia nobilis (Eichl) & 24 \\
\hline epicatequina $(\mathbf{2 1})$ & Luxemburgia nobilis (Eichl) & 24 \\
\hline 2,4,3'4'-OH-chalcona (3-hidroxiisoliquiritigenina,22) & Luxemburgia nobilis (Eichl) & 24 \\
\hline $2,4,4^{\prime}-\mathrm{OH}$-chalcona (isoliquiritigenina, 23 ) & Luxemburgia nobilis (Eichl) & 24 \\
\hline $\begin{array}{l}4^{\prime}, 5,7-\mathrm{OH}-\text { flavona- }\left(3^{\prime} \rightarrow 8^{\prime \prime}\right)-4^{\prime \prime \prime}, 5^{\prime \prime}, 7^{\prime \prime}-\mathrm{OH} \text { flavona. } \\
\text { (amentoflavona,11) }\end{array}$ & Luxemburgia nobilis (Eichl) & 24 \\
\hline $\begin{array}{l}4^{\prime}, 5,7-\mathrm{OH}-\text { flavona- }\left(3^{\prime} \rightarrow 6\right)-4^{\prime \prime \prime}, 5^{\prime \prime}, 7^{\prime \prime}-\mathrm{OH} \text { flavona } \\
\text { (robustaflavona, } 24)\end{array}$ & Luxemburgia nobilis (Eichl) & 24 \\
\hline $\begin{array}{l}4,2^{\prime}, 4^{\prime}-\mathrm{OH}-\text { chalcona- }\left(3 \rightarrow \mathrm{O} \rightarrow 4^{\prime \prime}\right)-2^{\prime \prime \prime}, 4^{\prime \prime \prime}-\mathrm{OH} \text { chalcona } \\
\text { (luxenchalcona,25) }\end{array}$ & Luxemburgia octandra. St. Hil. & 12 \\
\hline 2,4,3'4'-OH-chalcona (3-hidroxiisoliquiritigenina,22) & Luxemburgia octandra. St. Hil. & 12 \\
\hline $2,4,4^{\prime}-\mathrm{OH}$-chalcona (isoliquiritigenina,23) & Luxemburgia octandra. St. Hil. & 12 \\
\hline $4^{\prime}, 5,7-\mathrm{OH}-$ flavona- $\left(3^{\prime} \rightarrow \mathrm{O} \rightarrow 4^{\prime \prime \prime}\right)-5^{\prime \prime}, 7^{\prime \prime}-\mathrm{OH}$ flavanona $(\mathbf{2 0})$ & Luxemburgia octandra. St. Hil. & 12 \\
\hline 5,7,3', $4^{\prime}$-OH-8-C-glicopiranosil-flavona (26) & Luxemburgia octandra. St. Hil. & 36 \\
\hline 5,3,4-OH-7-OMe-8-C-glicopiranosil-flavona (27) & Luxemburgia octandra. St. Hil. & 36 \\
\hline calodenina B (28) & O. flava Schum e Thon & 35 \\
\hline flavumona A (29) & O. flava Schum e Thon & 35 \\
\hline flavumona B (30) & O. flava Schum e Thon & 35 \\
\hline calodenina $\mathrm{C}(\mathbf{3 1})$ & O. flava Schum e Thon & 35 \\
\hline lophirona A (32) & O. flava Schum e Thon & 35 \\
\hline $4^{\prime}, 5$-OMe-6,7-metilenodioxi-isoflavona (33) & O. flava Schum e Thon & 35 \\
\hline $\begin{array}{l}\text { 5-OH-7-OMe-flavona- }\left(4^{\prime} \rightarrow \mathrm{O} \rightarrow 8^{\prime \prime}\right)-5^{\prime \prime}, 4^{\prime \prime \prime \prime}-\mathrm{OH}-7^{\prime \prime \prime}-\mathrm{OMe} \\
\text { flavona }\left(7,7^{\prime \prime}-\mathrm{O} \text {-dimetillanaraflavona,34) }\right.\end{array}$ & O. hexasperma (St. Hil.) Bail & 18 \\
\hline $\begin{array}{l}4^{\prime}, 5,7-\mathrm{OH}-\text { flavona- }\left(6 \rightarrow 8^{\prime \prime}\right)-5^{\prime \prime}, 4^{\prime \prime \prime}-\mathrm{OH}-7^{\prime \prime}-\mathrm{OMe} \text { flavona. } \\
\left(7^{\prime \prime}-\mathrm{O}-\text { metilagatisflavona, } \mathbf{1 0}\right)\end{array}$ & O. hexasperma (St. Hil.) Bail & 18 \\
\hline $\begin{array}{l}4^{\prime}, 5,7-\mathrm{OH}-f l a v o n a\left(6 \rightarrow 8^{\prime \prime}\right)-4^{\prime \prime \prime}, 5,{ }^{\prime \prime} 7^{\prime \prime}-\mathrm{OH} \text { flavona, } \\
\text { (agastiflavona), }(\mathbf{3 5})\end{array}$ & O. hexasperma (St. Hil.) Bail & 18 \\
\hline epicatequina (21) & O. hexasperma (St. Hil.) Bail & 18 \\
\hline 6-C-glicopiranosil-luteolina (36) & O. hexasperma (St. Hil.) Bail & 18 \\
\hline 3-O-glicopiranosil-quercetina (37) & O. hexasperma (St. Hil.) Bail & 18 \\
\hline $2^{\prime \prime}-\mathrm{O}-\beta-\mathrm{D}$ - glicopiranosil-8-C- $\beta$-D-glicopiranosil luteolina (38) & O. hexasperma (St. Hil.) Bail & 18 \\
\hline
\end{tabular}


As flavonas e flavonóis (3-oxiflavonas) glicosilados são descritos como bioprodutos de espécies de Ouratea e Luxemburgia. São geralmente derivados da luteolina (5,7,3',4'-tetraidroxiflavona, 36) e de quercetina (3,5,7,3',4'-pentaidroxiflavona, 37), podendo ser, inclusive, metilados. A rutina (3-O-rutinosil quercetina, 19) foi isolada de Luxemburgia nobilis ${ }^{24}$ e de Ouratea semisserrata ${ }^{17}$. Das flores de Luxemburgia octandra foram isolados dois flavonóides 8- $C$-glicosila $(\mathbf{2 6}, \mathbf{2 7})$, Figura 2. A glicose aparece como o único carboidrato encontrado até o momento, entre os flavonóides $C$-glicosila isolados de espécies desses gêneros. Chama atenção a presença de isoflavonóides clorados, monoméricos, isolados somente de $O$. semisserata $(\mathbf{1 3}, \mathbf{1 4})$, além de chalconas presentes em duas espécies do gênero Luxemburgia $(\mathbf{2 2}, \mathbf{2 3})$.

\section{ASPECTOS FARMACOLÓGICOS E IMPORTÂNCIA ECONÔMICA DE ALGUMAS ESPÉCIES ESTUDADAS}

A avaliação dos trabalhos na literatura permitiu verificar a existência de vários estudos farmacológicos, tanto com frações de extratos brutos como com biflavonóides naturais e seus derivados isolados de espécies do gênero Ouratea e Luxemburgia.

Os biflavonóides 7"-O-metilagatisflavona (de O. hexasperma), a amentoflavona (de $O$. semisserrata) e o derivado acetilado da amentoflavona apresentaram atividade inibitória da DNA topoisomerase humana tipo I, potente atividade sobre a inibição do crescimento de células de carcinoma de Ehrlich, porém, apenas a agatisflavona apresentou atividade sobre a inibição da DNA topoisomerase humana tipo II- $\alpha$ e inibição de $42 \%$ do crescimento de células de leucemia humana K562 25,26 . As biflavonas $6 \rightarrow 6^{\prime \prime}$-begenkwanina e a 7,7"-O-dimetilagatisflavona isoladas de O. spectabilis apresentaram atividade inibitória sobre a enzima aldose redutase de cristalino bovino. $\mathrm{O}$ aumento da atividade dessa enzima está relacionado com a patogênese da maioria das complicações da diabetes, como cataratas, retinopatia, neuropatia ${ }^{16}$. $\mathrm{O}$ extrato hidroetanólico e a fração acetato de etila de $O$. semiserrata apresentaram efeito vasodilatador endotélio-dependente e atividade antiipertensiva in vitro, inibindo a conversão da enzima angiotensina $\mathrm{I}(\mathrm{ACE})^{27}$. O extrato aquoso de Ouratea $s p$., contendo proantocianidina, mostrou atividade antitumoral contra o carcinossarcoma de Walker 256 e sarcoma 180 em ratos ${ }^{28,29}$. O óleo extraído do extrato hexânico dos frutos de Ouratea parviflora apresentou atividades antibacteriana e antifúngica ${ }^{30}$. Os biflavonóides isolados de $O$. spectabilis, O. multiflora e $O$. parviflora mostraram inibição da produção de aflatoxina por Aspergillus flavus ${ }^{31}$.

\section{CONCLUSÕES}

Nesta revisão foi verificado que as substâncias mais significativas do gênero Ouratea são os biflavonóides com ligação interflavonoídica do tipo $\mathrm{C}-\mathrm{C}$, sendo o representante mais abundante o derivado da agatisflavona. Por outro lado o gênero Luxemburgia é caracterizado pela ligação do tipo C-O-C, cujo componente mais abundante é a $2^{\prime \prime}$-diidroochanaflavona, além das chalconas presentes apenas em espécies desse gênero, tanto na forma monomérica como nas bichalconas.

A distribuição do grupo de produtos naturais formados por acoplamento de duas unidades flavonoídicas nos gêneros Ouratea e Luxemburgia e o levantamento de suas propriedades biodinâmicas permitiram: caracterizá-los como marcadores quimiotaxonômicos para os referidos táxons, propor uma nomenclatura com notação para esse grupo de substâncias e evidenciar suas potencialidades farmacológicas.

Apesar dessas espécies não serem tão conhecidas na medicina popular, a freqüência das biflavonas é indicativa de ótimas perspectivas para se tornarem constituintes de medicamentos.

\section{REFERÊNCIAS}

1. Dahlgren, R. M. T.; Bot. J. Line Soc. 1980, 80, 91.

2. Joly, A. B. B.; Botânica: Introdução à Taxonomia Vegetal, 12 ${ }^{\mathrm{a}}$ ed., Cia Editora Nacional: São Paulo, 1988.

3. Barroso, G. M.; Sistemática de Angiospermas do Brasil, UFV: MG, 1986.

4. Tih, R. G.; Sondengam, B. L.; Martin, M. T.; Bodo, B.; Phytochemistry 1989, 28, 1557.

5. Messanga, B. B.; Kimbu, S. F.; Sondengam, B. L.; Bodo, B.; Phytochemistry 2002, 59, 435 .

6. Likhitwitayawuid, K.; Rungserchai, R.; Ruangrungsi, N.; Phadungcharoen, T.; Phytochemistry 2001, 56, 353.

7. Tih, A.; Martin, M. T.; Tih, R. G.; Vuidepot, I.; Sondengam, B. L.; Bodo, B.; Phytochemistry 1992, 31, 3595.

8. Lin, Y. M.; Flavin, M. T.; Schure, R.; Chen, F. C.; Sidwell, R.; Barnard, D. L.; Huffman, J. H.; Kern, E. R.; Planta Med. 1999, 65, 120.

9. Simões, C. M. O.; Schenkel, E. P.; Gusman, G.; Mello, J. C. P. de; Mentz, L. A; Petrovick, P. R.; Farmacognosia: Da Planta ao Medicamento, UFSC/ UFRGS: Florianópolis/Porto Alegre, 2001.

10. Chari, V. M.; Chen, F.; Chen, L.; Ilyas, M.; Lin, Y. C.; Lin, Y. M.; Neszmely, A.; Wagner, H.; Phytochemistry 1977, 16, 1273.

11. Dora, G.; Edwards, J. M.; J. Nat. Prod. 1991, 54, 796.

12. de Carvalho, M. G.; Alves, C. C. F.; Silva, K. G. S.; Eberlin, M. N.; Werle, A. A.; J. Braz. Chem. Soc. 2004, 15, 146.

13. Drewes, S. E.; Hudson, N. A.; Bates, R. B.; Linz, G. S.; Tetrahedron Lett. 1984, 25, 105.

14. Moreira, I. C.; Sobrinho, D. C.; de Carvalho, M. G.; Braz-Filho, R.; Phytochemistry 1994, 35, 1567.

15. Moreira, I. C.; de Carvalho, M. G.; Bastos A B. F.; Braz-Filho, R.; Phytochemistry 1999, 51, 833.

16. Felício, J. D.; Gonçalez, E.; Braggio, M. M.; Constantino, L.; Albasini, A; Lins, A. P.; Planta Med. 1995, 61, 217

17. Velandia, J. R.; de Carvalho, M. G.; Braz-Filho, R.; Werle A. A.; Phytochem. Anal. 2002, 13, 283.

18. Daniel, J. F. de S.; de Carvalho, M. G.; Cardoso, R. da S.; Agra, M. de F.; Eberlin, M. N.; J. Braz. Chem. Soc. 2005, 16, 634.

19. Pegnyemb, D. E.; Tih, R. G.; Sondengam, B. L.; Blond, A.; Bodo, B.; Phytochemistry 2001, 57, 579.

20. Felicio, J. D.; Rossi, M. H.; Braggio, M. M.; Gonzalez, E.; Pak, A.; Cordeiro, I.; Felício, R. C.; Biochem. Syst. Ecol. 2004, 32, 79

21. de Carvalho, M. G.; Carvalho, G. J. A. de; Braz-Filho, R.; J. Braz. Chem. Soc. 2000, 11, 143

22. de Carvalho, M. G.; Oliveira, M. C. C.; Werle, A. A.; J. Braz. Chem. Soc. 2002, 11, 232

23. Velandia, J. R.; de Carvalho M. G.; Braz-Filho, R.; Quim. Nova 1998, 21, 397.

24. Oliveira, M. C. C.; de Carvalho, M. G.; Silva, C. J.; Werle, A. A. ; J. Braz. Chem. Soc. 2002, 13, 119.

25. Grynberg, N. F.; de Carvalho, M. G.; Velandia, J. R.; Oliveira, M. C.; Moreira, I. C.; Braz-Filho, R.; Echevarria, A.; Braz. J. Med. Biol. Res. 2002, 35,819 .

26. de Carvalho, M. G.; Velandia, J. R.; de Oliveira, J. C. C.; Echevarria, A.; BrazFilho, R.; Grynberg, N. F. Em Phytochemical and Pharmacologiy II of the Series "Recent Progress in Medicinal Plants", Majumdar, D. K.; Govil, J. N.; Singh, V. K., eds.; SCI Tech Publishing LLC: Texas, 2002, vol. 8, p. 77-92.

27. Cortes, S. F.; Valadares, M. Y.; Oliveira, A B. de; Lemos. S. V.; Barbosa, M. P. T.; Braga, F. C.; Planta Med. 2002, 68, 412.

28. Sampaio, M. P.; Oliveira, M. M. de; An. Acad. Bras. Cienc. 1975, 47, 149.

29. Oliveira, M. M. de; Sampaio, M. P.; Simon, F.; Gilbert, B.; Mors, W. B.; An. Acad. Bras. Ciênc. 1972, 44, 41.

30. Marcol, P. Q.; Lima, E. O.; Maia, R. F.; Xavier, L. F.; Chemical Abstract 1988, 108, 399.

31. Gonçalez, E.; Felício, J. D.; Pinto, M. M.; Braz. J. Med. Biol. Res. 2001, $34,1453$.

32. Monache, F. D.; Albuquerque, I. L. de; Ferrari, F.; Betollo, G. B. M.; Tetrahedron Lett. 1967, 84, 211.

33. Gartlan, S.; Mckey, D. B.; Waterman, P. G.; Mbi, C. N.; Struhsaker, T. T.; Biochem. Syst. Ecol. 1980, 8, 401.

34. Felício, J. D.; Rossi, M. H.; Park, H. R.; Gonçalez, E.; Braggio, M. M.; David, J. M.; Cordeiro, I.; Fitoterapia 2001, 72, 453.

35. Mbing, J. N.; Pegnyemb, D. E.; Tih, R. G.; Ghogomu T. R.; Sondemgam, B. L.; Blond, A.; Bodo, B.; Phytochemistry 2003, 63, 427.

36. Alves, C. C. F.; Tese de Doutorado, Universidade Federal Rural do Rio de Janeiro, Brasil, 2003. 